\title{
DOSES DE ENXOFRE E TEMPO DE INCUBAÇÃO NA DINÂMICA DE FORMAS DE ENXOFRE NO SOLO ${ }^{(1)}$
}

\author{
Sandra Cátia Pereira Uchôa ${ }^{(2)}$, Víctor Hugo Alvarez V. ${ }^{(3)}$ \& Francisco \\ Morel Freire ${ }^{(4)}$
}

\begin{abstract}
RESUMO
A disponibilidade de enxofre (S) depende da dinâmica de suas formas no solo. Visando estudar o equilíbrio entre as reservas de $\mathrm{S}$ lábil e $\mathrm{S}$ não lábil, amostras de 10 solos foram incubadas com cinco doses de sulfato de cálcio $(0,20,50,80$ e $120 \mathrm{mg} \mathrm{dm}^{-3}$ de $S$ ), durante $1,8,15,30,60$ e 90 d. Para a determinação do $S$ lábil, utilizou-se membrana de resina de troca aniônica em forma de lâmina. $O$ S extraído com $48 \mathrm{~h}$ de agitação foi denominado de enxofre rapidamente lábil (SRL). $O$ teor obtido com tempo de agitação superior a $48 \mathrm{~h}$ foi denominado de enxofre lentamente lábil e o não lábil (SLL+SNL), pela diferença entre o S do solo (extraído após calcinação) e SRL. A resistência que o solo oferece à passagem do $\mathbf{S}$ da reserva do SRL para a do SLL+SNL foi denominada de capacidade-tampão do S lábil (CTSL), sendo obtida pelo inverso do coeficiente angular das equações lineares do teor de SRL em função do teor de SLL+SNL. Constatou-se que tempo de incubação (reatividade) e as doses de $\mathrm{S}$, na forma de sulfato de cálcio, influenciam a dinâmica do S entre as reservas de SRL e SLL+SNL. A CTSL é sensível às mudanças no equilíbrio entre as reservas de $\mathrm{S}$ no solo. $\mathrm{O}$ aumento da CTSL dos solos relacionou-se, principalmente, com a capacidade máxima de adsorção de sulfato, teor de carbono orgânico, goethita e gibbsita da fração argila dos solos estudados.
\end{abstract}

Termos de indexação: enxofre lábil, enxofre não lábil, fixação, lâmina de resina de troca aniônica, capacidade tampão do enxofre lábil.

\footnotetext{
(1) Parte da Tese de Doutorado do primeiro autor, apresentado ao Programa de Pós-graduação em Solos e Nutrição de Plantas, Universidade Federal de Viçosa. Recebido para publicação em 6 de setembro de 2011 e aprovado 12 de março de 2013.

(2) Professora Associada IV, Departamento de Solos e Engenharia Agrícola, Universidade Federal de Roraima-UFRR. Rodovia BR 174, Campus do Cauamé. CEP 69314-080 Boa Vista (RR). E-mail: scpuchoa@dsi.uffr.br

(3) Professor Titular, Departamento de Solos, UFV. Bolsista CNPq. CEP 36571-000 Viçosa (MG). E-mail: vhav@ufv.br

(4) Pesquisador EPAMIG/CPACD. Rua Teófilo Otoni, 702/501. CEP 35700-007 Sete Lagoas (MG). E-mail: freire@embrapa.br
} 


\title{
SUMMARY: EFFECT OF SULPHUR RATES AND INCUBATION TIME ON THE DYNAMICS OF SULPHUR FORMS IN SOIL
}

\begin{abstract}
Sulphur availability depends on the dynamics of its soil forms. An experiment was carried out to evaluate the effects of adding sulphur doses (0, 20,50, 80, and $120 \mathrm{mg} \mathrm{dm}^{-3}$ of S), as calcium sulphate, and the incubation time $(1,8,15,30,60$, and $90 \mathrm{~d})$ on the equilibrium between labile and non-labile sulphur reserves, in 10 soils. An anion exchange resin membrane was used to determine labile sulphur. A shaking period of $48 \mathrm{~h}$ (of water, soil and resin mixture) extracted a greater part of labile sulphur, which was termed quickly-labile sulphur (QLS). The slowly-labile sulphur plus the non-labile sulphur (SLS+NLS) were determined by the difference between the soil sulphur by calcination and the SRL. The soil resistance against passing the $S R L$ reserve to $S L L+S N L$ was named labile sulphur buffering capacity $(L S B C)$, computed by the inverse angular coefficient of the linear equations between SRL and SLL+SNL contents. In general, it was observed that incubation time (reactivity) and $S$ doses, in the form of calcium sulphate, affect the sulphur dynamics between $Q L S$ reserves and $S L S+N L S$. The $L S B C$ is sensitive to changes in the balance between sulfur reserves in the soil. The increase in $L S B C$ in the soil was mainly related to the maximum adsorption capacity of sulphate (MACS), and contents of organic carbon, goethite and gibbsite in the clay fraction of the soils.
\end{abstract}

Index terms: labile sulphur, non-labile sulphur, anionic exchange resin sheet, labile sulphur buffering capacity.

\section{INTRODUÇÃO}

A disponibilidade de enxofre (S) em termos físicoquímicos pode ser explicada pela inter-relação entre os fatores quantidade (Q), que mede a reserva lábil; intensidade (I), que avalia a concentração desse elemento na solução do solo; e capacidade-tampão (CT), que estima a capacidade de reposição do S lábil para a solução. Além dessas inter-relações, também existe reposição, mais lenta, entre as formas lábeis e não lábeis (Alvarez V., 1988; Uchôa et al., 2003).

Blair (1979) sugeriu que a adsorção é o principal mecanismo controlador da disponibilidade do S para as plantas, podendo ocorrer em superfície carregada positiva (adsorção eletrostática) ou negativamente (adsorção química ou específica). Rajan (1979) propôs que, em uma superfície neutra ou negativa, o $\mathrm{SO}_{4}{ }^{2-}$ é adsorvido como monodentado, sendo para isso necessário deslocar um ligante aquo ou ligante hidroxila. Numa superfície positiva, o $\mathrm{SO}_{4}{ }^{2-}$ é adsorvido como bidentado, formando estrutura anelar de seis membros que, segundo Hingston et al. (1974), favorece a irreversibilidade, ou seja, formas não lábeis. Adams \& Rawajfih (1977) sugeriram a precipitação e dissolução da basalunita e alunita e de sais análogos de Fe como mecanismos plausíveis para os fenômenos de sorção de $\mathrm{SO}_{4}{ }^{2-}$ em solos ácidos.

A capacidade relativa de adsorção de $\mathrm{SO}_{4}{ }^{2-}$ pelos vários constituintes do solo tem sido demonstrada em vários trabalhos. Tem-se verificado que os óxidos de $\mathrm{Al}$ e $\mathrm{Fe}$ são mais adsortivos que as argilas do tipo 1:1 que, por sua vez, adsorvem mais que as argilas do tipo 2:1 (Ensminger, 1954; Chao et al., 1964; Aylmore et al., 1967; Ajwa \& Tabatabai, 1995). Quanto aos óxidos, os de $\mathrm{Al}$ tendem a ser mais adsortivos que os de Fe (Ensminger, 1954; Chao et al., 1964). Alves \&
Lavorenti (2004) observaram elevada correlação entre adsorção de sulfato em solos intemperizados, contendo óxidos de $\mathrm{Fe}$ e $\mathrm{Al}$ de baixa cristalinidade. Conforme Kimsey Jr. et al. (2005), a cristalinidade dos óxidos de $\mathrm{Fe}$ e Al influenciou na dessorção de sulfato, sendo menor sua disponibilidade na solução de solos com óxidos de menor cristalinidade.

Os efeitos da matéria orgânica do solo na retenção do $\mathrm{SO}_{4}{ }^{2-}$ são contraditórios. Alguns trabalhos têm evidenciado relação direta na retenção por causa da formação de ponte de $\mathrm{Ca}, \mathrm{Al}$ e $\mathrm{Fe}$ (Couto et al., 1979; Barton et al., 1999), ao passo que outros têm evidenciado relação negativa da adsorção de $\mathrm{SO}_{4}{ }^{2-}$ pelos coloides orgânicos (Patil et al., 1989; Strahm \& Harrison, 2007) e inconclusivas (Kimsey Jr. et al., 2005).

A literatura evidencia, principalmente por meio dos processos de adsorção, a formação das reservas lábeis e não lábeis de $\mathrm{S}$ no solo. Como a reversibilidade entre essas formas constitui-se em um processo contínuo, separá-las, identificá-las e quantificá-las é objeto dos métodos de extração. Uchôa et al. (2003) sugeriram um método que define uma fração do $\mathrm{S}$ lábil, denominada de $\mathrm{S}$ rapidamente lábil, utilizando membrana de resina de troca aniônica e agitação contínua durante $48 \mathrm{~h}$. Salim et al. (2004) propuseram um modelo de multirreações para a reatividade do $\mathrm{SO}_{4}{ }^{2-}$ em solos de floresta,em que consideraram: dois reservatórios rapidamente lábeis, cuja relação com o fator intensidade é governada por reações de cinética não linear; um reservatório de reversibilidade lenta em equilíbrio com um dos reservatórios rapidamente lábeis; e um reservatório irreversível.

Considera-se que fatores relacionados ao manejo do solo, por meio da adubação com S e do tempo de reação do adubo com os coloides do solo, levam à 
formação de uma reserva não lábil do $\mathrm{S}$, que possivelmente controla as perdas desse nutriente por lixiviação e influencia na sua disponibilidade e de outros nutrientes às plantas. A questão básica fundamenta-se em torno dessa dinâmica entre S lábil e não lábil, no que concerne a fatores e propriedades do solo que interferem no equilíbrio entre as reservas.

Mediante o exposto, objetiva-se com este trabalho estudar o equilíbrio entre as reservas do S lábil e do S não lábil em amostras de solos submetidas à adição de diferentes doses de $\mathrm{S}$ e tempos de incubação.

\section{MATERIAL E MÉTODOS}

Um experimento foi montado em condições de casa de vegetação, empregando-se 10 solos com ampla variação nas propriedades químicas, físicas, físicoquímicas e mineralógicas, pertencentes às classes: Neossolo Quartzarênico; Latossolo Vermelho-Amarelo; Latossolo Vermelho; e Latossolo Amarelo. As amostras dos solos da camada superficial de $0-20 \mathrm{~cm}$ de profundidade foram coletadas nos Estados de Minas Gerais e Espírito Santo. A caracterização das subamostras dos solos é apresentada nos quadros 1 e 2 .

Para a condução do experimento, as amostras dos 10 solos foram simultaneamente incubadas em sacos plásticos fechados, com cinco doses de $\mathrm{S}(0,20,50,80$ e $120 \mathrm{mg} \mathrm{dm}^{-3} \mathrm{de} \mathrm{S}$ ), utilizando o reagente $\mathrm{CaSO}_{4} \cdot 2 \mathrm{H}_{2} \mathrm{O}$ p.a., durante seis tempos de incubação $(1,8,15,30,60$ e 90 d). A combinação dos fatores em estudo proporcionou o esquema fatorial $10 \times 5 \times 6$, correspondendo a 10 solos, cinco doses de $\mathrm{S}$ e seis tempos de incubação. Os tratamentos assim obtidos foram distribuídos no delineamento de blocos casualizados, com três repetições. A parcela consistiu de saco plástico de polietileno preto com $500 \mathrm{~cm}^{3}$ de solo.

Durante a fase de incubação, manteve-se a umidade das amostras próxima a $80 \%$ da capacidade de campo por meio de pesagens semanais. Encerrada a fase de incubação, as amostras foram secas e peneiradas a $2 \mathrm{~mm}$ de malha.

Para determinar o efeito das doses de S e do tempo de incubação no equilíbrio entre as reservas de $\mathrm{S}$, foram avaliados o $\mathrm{S}$ rapidamente lábil (SRL) e o enxofre lentamente lábil mais o enxofre não lábil (SLL+SNL). O SRL e SLL+SLN foram definidos por meio de métodos preconizados por Uchôa et al. (2003), em que se definiu tempo de agitação de $48 \mathrm{~h}$ e emprego de membrana de troca aniônica na extração do SRL. O S lentamente lábil mais o não lábil (SLL+SNL) foram obtidos pela diferença entre o S do solo (S-so), estabelecido por calcinação (Bardsley \& Lancaster, 1960), e o S rapidamente lábil (SRL).

Na determinação do S lábil, utilizou-se membrana de resina de troca aniônica do tipo AR 103-QDP (Ionics,
Watertown, MA) na forma de lâmina com 0,57 mm de espessura, fabricada com malha reforçada de copolímeros de vinil e embebida em amônio quaternário (Ionics, 1987). Os procedimentos de preparo, précondicionamento e recuperação da membrana seguiram os recomendados por Uchôa et al. (2003).

Dentro de cada tempo de incubação e para cada dose de $\mathrm{S}$ adicionada, foram determinados o teor de SRL e o de SLL+SNL. A relação entre SRL e SLL+SNL foi modelada por meio de regressão linear. O poder tampão para a reserva do S lábil, denominado de capacidade tampão do enxofre lábil (CTSL), definida como a relação entre variação da concentração do $\mathrm{SLL}+\mathrm{SNL}$ e do SRL $(\mathrm{CTSL}=\Delta \mathrm{SLL}+\mathrm{SNL} / \Delta \mathrm{SRL})$, foi obtido utilizando analogia ao fator capacidade-tampão $(\mathrm{CT}=\Delta \mathrm{Q} / \Delta \mathrm{I})$ (Novais \& Smyth, 1999). Essa propriedade físico-química quantifica a concentração do S adicionado, que passa ao compartimento do SLL + SNL para cada unidade que fica no reservatório do $\mathrm{SRL}$, cuja unidade é $\mathrm{mg} \mathrm{dm} / 3 / \mathrm{mg} \mathrm{dm}^{-3}$. A CTSL foi separada em cinco classes. O limite entre as classes foi estabelecido pela relação entre a diferença do maior e menor valor de CTSL média e o número de intervalos entre a classe muito baixa e muito alta.

A significância das análises de variância e de regressão foi com base no teste $\mathrm{F}(\mathrm{p}<0,05)$. O teste "t" foi utilizado para testar os coeficientes da regressão no mesmo nível de probabilidade. O teor de SRL em função do de SLL+SNL ajustou-se melhor ao modelo linear, tendo sido considerado para a seleção do modelo o maior $R^{2}$ e a significância dos coeficientes de regressão. As equações tiveram sua variável independente calculada ao subtrair determinado valor, variável para cada solo e tempo de incubação, para se obter valor de SRL maior que zero $(\hat{y}>0)$.

\section{RESULTADOS E DISCUSSÃO}

O efeito das doses de Se dos tempos de incubação foi significativo pelo teste $\mathrm{F}(\mathrm{p}<0,05)$, demonstrando que os fatores estudados interferiram na dinâmica entre as reservas. No quadro 3, verifica-se que o aumento das doses de $\mathrm{S}$ e do tempo de incubação promoveram partição do S entre as reservas de SRL e SLL+SNL. Considerando o tempo de incubação de $90 \mathrm{~d}$ e a maior dose de S, a partição foi destacada no LVA-PA, permanecendo na reserva SRL aproximadamente 60

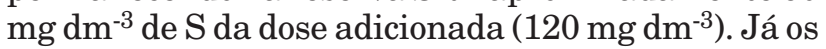
solos RQ-CV, LV-CV, LA-AR e LV-TM apresentaram baixa partição entre as reservas; acima de $100 \mathrm{mg} \mathrm{dm}^{-3}$ de S permaneceram na SRL. Os demais solos evidenciaram partição intermediária. A partição dos nutrientes entre as fases sólida e solúvel é importante fator controlador do transporte do nutriente, bem como da sua disponibilidade para as plantas e da lixiviação (Barrow, 1987). Compreender a influência das propriedades do solo na partição das quantidades de 
Quadro 1. Características químicas, físicas e físico-químicas das amostras dos solos estudados

\begin{tabular}{|c|c|c|c|c|c|c|c|c|c|c|}
\hline \multirow{2}{*}{ Característica } & \multicolumn{10}{|c|}{ Solo } \\
\hline & RQ-CV ${ }^{(1)}$ & LVA-TM ${ }^{(2)}$ & $\mathbf{L V}-\mathrm{CV}^{(3)}$ & $\mathbf{L A}-\mathbf{A} \mathbf{R}^{(4)}$ & LV-TM ${ }^{(5)}$ & LVA-UB1 $^{(6)}$ & LVA-UB2 ${ }^{(7)}$ & LV-SL ${ }^{(8)}$ & LVA-PR $^{(9)}$ & LVA-PA $^{(10)}$ \\
\hline $\mathrm{pH}\left(\mathrm{H}_{2} \mathrm{O}\right)(1: 2.5)$ & 5,05 & 4,64 & 4,97 & 4,40 & 4,99 & 5,00 & 5,34 & 4,38 & 4,70 & 4,64 \\
\hline P-solo $\left(\mathrm{mg} \mathrm{dm}^{-3}\right)^{(11)}$ & 64,40 & 115,60 & 242,60 & 64,40 & 41,00 & 363,00 & 298,00 & 934,00 & 228,00 & 439,60 \\
\hline P-disp. $\left(\mathrm{mg} \mathrm{dm}^{-3}\right)^{(12)}$ & 2,90 & 0,70 & 1,30 & 0,30 & 0,20 & 0,20 & 0,20 & 1,30 & 0,50 & 0,20 \\
\hline P-rem $\left(\mathrm{mg} \mathrm{L}^{-1}\right)^{(13)}$ & 37,10 & 38,10 & 21,10 & 29,90 & 29,90 & 4,80 & 3,40 & 3,20 & 4,60 & 1,80 \\
\hline S-Solo $\left(\mathrm{mg} \mathrm{dm}^{-3}\right)^{(14)}$ & 24,90 & 28,73 & 50,73 & 72,15 & 19,13 & 96,75 & 72,53 & 113,70 & 72,98 & 98,40 \\
\hline $\mathrm{K}$-disp. $\left(\mathrm{mg} \mathrm{dm}^{-3}\right)^{(15)}$ & 49,00 & 19,00 & 45,00 & 12,00 & 11,00 & 30,00 & 31,00 & 31,00 & 39,00 & 72,00 \\
\hline $\mathrm{Ca}^{2+}\left(\mathrm{cmol}_{\mathrm{c}} \mathrm{dm}^{-3}\right)^{(15)}$ & 0,18 & 0,00 & 0,24 & 0,19 & 0,00 & 0,00 & 0,08 & 0,94 & 0,10 & 0,19 \\
\hline $\mathrm{Mg}^{2+}\left(\mathrm{cmol}_{\mathrm{c}} \mathrm{dm}^{-3}\right)^{(15)}$ & 0,17 & 0,04 & 0,42 & 0,10 & 0,03 & 0,06 & 0,09 & 0,15 & 0,13 & 0,16 \\
\hline $\mathrm{Al}^{3+}\left(\mathrm{cmol}_{\mathrm{c}} \mathrm{dm}^{-3}\right)^{(15)}$ & 1,13 & 0,75 & 1,41 & 1,50 & 0,75 & 1,08 & 0,90 & 1,74 & 0,89 & 0,52 \\
\hline $\mathrm{H}+\mathrm{Al}\left(\mathrm{cmol}_{\mathrm{c}} \mathrm{dm}^{-3}\right)^{(16)}$ & 4,64 & 3,17 & 5,22 & 3,64 & 2,48 & 8,28 & 7,89 & 12,84 & 7,89 & 8,66 \\
\hline $\mathrm{SB}\left(\mathrm{cmol}_{\mathrm{c}} \mathrm{dm}^{-3}\right)$ & 0,48 & 0,09 & 0,78 & 0,32 & 0,06 & 0,14 & 0,25 & 1,17 & 0,24 & 0,54 \\
\hline $\mathrm{CTC}_{\text {efet. }}\left(\mathrm{cmol}_{\mathrm{c}} \mathrm{dm}^{-3}\right)$ & 1,61 & 0,84 & 2,19 & 1,82 & 0,081 & 1,22 & 1,15 & 2,91 & 1,13 & 1,06 \\
\hline $\mathrm{CTC}_{\mathrm{pH} 7}\left(\mathrm{cmol}_{\mathrm{c}} \mathrm{dm}^{-3}\right)$ & 6,25 & 4,01 & 7,41 & 5,46 & 3,29 & 9,50 & 9,04 & 15,75 & 9,02 & 9,72 \\
\hline $\mathrm{V}(\%)$ & 7,68 & 2,24 & 10,50 & 5,86 & 1,82 & 1,47 & 2,77 & 7,44 & 2,66 & 5,56 \\
\hline $\left.\mathrm{CO}(\mathrm{dag} \mathrm{kg})^{-1}\right)^{(17)}$ & 0,51 & 0,91 & 0,82 & 0,43 & 0,52 & 2,13 & 2,28 & 3,67 & 2,11 & 2,85 \\
\hline $\mathrm{CMAS}_{1}\left(\mathrm{mg} \mathrm{dm}^{-3} \mathrm{de} \mathrm{S}^{(18)}\right.$ & 11 & 12 & 63 & 64 & 67 & 188 & 188 & 199 & 215 & 279 \\
\hline 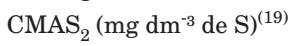 & - & 7 & 8 & 111 & 21 & 201 & 156 & 84 & 197 & 350 \\
\hline CMAF (mg dm ${ }^{-3}$ de P) ${ }^{(20)}$ & 397 & 335 & 659 & 453 & 434 & 1.887 & 1.673 & 1.757 & 1.728 & 1.936 \\
\hline $\mathrm{CC}\left(\text { dag kg }{ }^{-1}\right)^{(21)}$ & 14,80 & 15,40 & 18,90 & 18,00 & 18,00 & 35,40 & 33,40 & 36,70 & 35,40 & 37,30 \\
\hline Dens. do solo $\left(\mathrm{kg} \mathrm{dm}^{-3}\right)^{(22)}$ & 1,30 & 1,39 & 1,25 & 1,31 & 1,38 & 0,96 & 0,98 & 0,96 & 0,94 & 1,0 \\
\hline Areia grossa $(\%)^{(23)}$ & 58,0 & 50,0 & 52,0 & 46,0 & 51,0 & 15,0 & 11,0 & 8,0 & 3,0 & 18,0 \\
\hline Areia fina $(\%)^{(23)}$ & 29,0 & 36,0 & 23,0 & 17,0 & 30,0 & 6,0 & 6,0 & 7,0 & 8,0 & 4,0 \\
\hline Silte $(\%)^{(23)}$ & 1,0 & 2,0 & 2,0 & 2,0 & 3,0 & 19,0 & 16,0 & 13,0 & 21,0 & 24,0 \\
\hline Argila (\%) $)^{(23)}$ & 12,0 & 12,0 & 23,0 & 35,0 & 16,0 & 60,0 & 67,0 & 72,0 & 68,0 & 54,0 \\
\hline
\end{tabular}

(1) Neossolo Quartzarênico-Campina Verde; ${ }^{(2)}$ Latossolo Vermelho-Amarelo-Três Marias; ${ }^{(3)}$ Latossolo Vermelho-Campina Verde; (4) Latossolo Amarelo-Aracruz; ${ }^{(5)}$ Latossolo Vermelho-Três Marias; ${ }^{(6)}$ Latossolo Vermelho-Amarelo-Uberaba (Amostra 1); ${ }^{(7)}$ Latossolo Vermelho-Amarelo-Uberaba (Amostra 2); ${ }^{(8)}$ Latossolo Vermelho-Sete Lagoas; ${ }^{(9)}$ Latossolo Vermelho-Amarelo-Paracatu; ${ }^{(10)}$ Latossolo Vermelho-Amarelo-Patrocínio; ${ }^{(11)}$ Ataque $\mathrm{HNO}_{3} / \mathrm{HClO}_{4}$; ${ }^{(12)}$ Extrator Mehlich-1 (Defelipo \& Ribeiro, 1981); ${ }^{(13)} \mathrm{P}$ remanescente (Alvarez V. et al., 2000); ${ }^{(14)} \mathrm{S}$ do solo obtido por calcinação (Bardsley \& Lancaster, 1960); ${ }^{(15)}$ Extrator KCl 1 mol/L (Defelipo \& Ribeiro, 1981); ${ }^{(16)}$ Extrator $\mathrm{Ca}(\mathrm{OAc})_{2}$ 0,5 mol L-1, pH 7,0 (Defelipo \& Ribeiro, 1981); ${ }^{(17)}$ CO, Método Walkley-Black (Defelipo \& Ribeiro, 1981); ${ }^{(18)}$ Capacidade Máxima de Adsorção de Sulfato (Alvarez V. et al., 2001); ${ }^{(19)}$ Capacidade Máxima de Adsorção de Sulfato nos solos,após o ataque com $\mathrm{H}_{2} \mathrm{O}_{2} 30$ vol. (Alvarez V. et al., 2001); ${ }^{(20)}$ Capacidade Máxima de Adsorção de Fosfato (Olsen \& Watanabe, 1957), com as doses definidas de acordo com o valor do P remanescente (Alvarez V. et al., 2000); ${ }^{(21)}$ Capacidade de Campo - Método da coluna (Fernandes, 1967); ${ }^{(22)}$ Método da proveta; e ${ }^{(23)}$ Método da pipeta (Moura Filho, 1964).

ânions entre as fases do solo é importante para predizer seus fluxos em resposta direta à adubação $\mathrm{e}$ indireta a outros manejos na paisagem (Strahm \& Harrison, 2007).

A partição entre as reservas SRL e SLL+SLN dos solos foi influenciada pela capacidade máxima de adsorção de sulfato - CMAS (Quadro 1). O grupo de solos com menor partição de S (RQ-CV, LV-CV, LAAR e LV-TM) apresentou CMAS inferior a $67 \mathrm{mg} \mathrm{dm}^{-3}$. Com exceção do LVA-TM, os demais solos, com partição intermediária e alta, apresentaram CMAS superior a $188 \mathrm{mg} \mathrm{dm}^{-3}$. O LVA-TM, embora com baixa CMAS (12 $\mathrm{mg} \mathrm{dm}^{-3}$ ), encontra-se no grupo de solos que apresentou partição intermediária. É possível que o teor de matéria orgânica tenha incrementado o número de sítios de fixação do sulfato, favorecendo a dinâmica em favor da reserva SLL+SNL.

Pela CMAS também se explica a elevada reserva de SLL+SNL, superior a $90 \mathrm{mg} \mathrm{dm}^{-3}$ de S, aos $90 \mathrm{~d}$ de incubação e na maior dose de S, nos solos LVA-UB1, LVA-UB2, LV-SL, LVA-PR e LVA-PA (Quadros 1 e 3), que por sua vez decorre, provavelmente, dos elevados teores dos óxidos de $\mathrm{Fe}$ e $\mathrm{Al}$ presentes na fração argila (Quadro 2). Vários estudos têm demonstrado a elevada CMAS nos óxidos de $\mathrm{Fe}$ e $\mathrm{Al}$. Óxidos de $\mathrm{Fe}$ e $\mathrm{Al}$ de baixa cristalinidade foram significativamente correlacionados com adsorção de $\mathrm{SO}_{4}{ }^{2-}$ (Alves \& Lavorenti, 2004). Solos com quantidades variáveis de óxidos cristalinos adsorvem de 300 a $11.000 \mathrm{~kg} \mathrm{ha}^{-1} \mathrm{de} \mathrm{SO}_{4}{ }^{2-}$ (Barton et al., 1994; Camps Aberstain et al., 2002).

Na figura 1, tem-se a estimativa da variação do SRL em função de SLL+SNL para cada tempo de incubação. Por meio da variação do coeficiente angular das funções, visualiza-se a dinâmica que se deu entre as reservas ao longo do tempo de incubação. $\mathrm{O}$ inverso do coeficiente angular foi definido como capacidade-tampão do enxofre lábil - CTSL (Figura 1 e Quadro 4). 
Quadro 2. Composição mineralógica da fração argila (Caulinita -Ct; Gibbsita -Gb; e Goethita -Gt) calculada a partir dos teores de óxidos obtidos pelo ataque sulfúrico, valores de Ki e Kr e relações dos óxidos nas amostras dos solos estudados

\begin{tabular}{|c|c|c|c|c|c|c|c|c|c|c|c|c|}
\hline \multirow{3}{*}{ Solo } & \multicolumn{5}{|c|}{ Ataque Sulfúrico ${ }^{(11)}$} & \multicolumn{3}{|c|}{ Comp. Mineralógica ${ }^{(12)}$} & \multirow{3}{*}{$\mathbf{K i}$} & \multirow{3}{*}{$\mathbf{K r}$} & \multicolumn{2}{|c|}{ Rel. Molecular } \\
\hline & \multirow{2}{*}{$\mathrm{SiO}_{2}$} & \multirow{2}{*}{$\mathbf{A l}_{2} \mathbf{O}_{3}$} & \multirow{2}{*}{$\mathrm{Fe}_{2} \mathrm{O}_{3}$} & \multirow{2}{*}{$\mathrm{TiO}_{2}$} & \multirow{2}{*}{$\overline{\mathbf{P}_{2} \mathbf{O}_{5}}$} & \multirow{2}{*}{$\mathbf{C t}$} & \multirow{2}{*}{$\mathbf{G b}$} & \multirow{2}{*}{ Gt } & & & \multirow{2}{*}{$\begin{array}{l}\mathrm{Al}_{2} \mathrm{O}_{3} \\
\mathrm{Fe}_{2} \mathrm{O}_{3} \\
\end{array}$} & \multirow{2}{*}{$\begin{array}{r}\mathrm{SiO}_{2} \\
\mathrm{Fe}_{2} \mathrm{O}_{3} \\
\end{array}$} \\
\hline & & & & & & & & & & & & \\
\hline & & & & - dag & & & & & & & & \\
\hline $\mathrm{RQ}-\mathrm{CV}^{(1)}$ & 4,6 & 3,57 & 2,69 & 0,54 & 0,02 & 8,6 & - & 3,7 & 2,20 & 1,48 & 2,08 & 4,56 \\
\hline LVA-TM ${ }^{(2)}$ & 3,8 & 8,16 & 2,50 & 0,99 & 0,01 & 9,8 & 1,6 & 2,8 & 1,52 & 1,18 & 3,47 & 5,29 \\
\hline $\mathrm{LV}-\mathrm{CV}^{(3)}$ & 7,7 & 6,63 & 6,89 & 2,30 & 0,06 & 16,5 & - & 6,7 & 1,97 & 1,18 & 1,51 & 2,90 \\
\hline $\mathrm{LA}-\mathrm{AR}^{(4)}$ & 13,8 & 12,23 & 2,99 & 1,93 & 0,01 & 29,8 & 1,4 & 2,2 & 1,83 & 1,60 & 6,94 & 12,69 \\
\hline LV-TM ${ }^{(5)}$ & 4,5 & 5,10 & 2,30 & 0,88 & 0,01 & 8,3 & 7,3 & 2,9 & 0,80 & 0,67 & 5,11 & 4,08 \\
\hline LVA-UB1 $^{(6)}$ & 8,8 & 36,32 & 5,89 & 2,25 & 0,07 & 35,4 & 35,4 & 9,6 & 0,85 & 0,73 & 5,83 & 4,98 \\
\hline LVA-UB2 ${ }^{(7)}$ & 16,4 & 32,75 & 8,78 & 1,88 & 0,08 & 19,0 & 19,0 & 5,5 & 0,41 & 0,37 & 9,66 & 3,98 \\
\hline LV-SL ${ }^{(8)}$ & 24,7 & 27,78 & 11,68 & 1,03 & 0,21 & 14,3 & 10,1 & 14,3 & 1,51 & 1,19 & 3,72 & 5,63 \\
\hline LVA-PR ${ }^{(9)}$ & 23,5 & 36,07 & 8,18 & 1,23 & 0,06 & 50,7 & 24,3 & 9,7 & 1,11 & 0,97 & 6,90 & 7,66 \\
\hline LVA-PA ${ }^{(10)}$ & 6,0 & 28,93 & 13,67 & 0,78 & 0,09 & 14,4 & 30,4 & 17,7 & 0,44 & 0,33 & 2,85 & 1,26 \\
\hline
\end{tabular}

(1) Neossolo Quartzarênico-Campina Verde; ${ }^{(2)}$ Latossolo Vermelho-Amarelo-Três Marias; ${ }^{(3)}$ Latossolo Vermelho-Campina Verde; ${ }^{(4)}$ Latossolo Amarelo-Aracruz; ${ }^{(5)}$ Latossolo Vermelho-Três Marias; ${ }^{(6)}$ Latossolo Vermelho-Amarelo-Uberaba (Amostra 1); (7) Latossolo Vermelho-Amarelo-Uberaba (Amostra 2); ${ }^{(8)}$ Latossolo Vermelho-Sete Lagoas; ${ }^{(9)}$ Latossolo Vermelho-AmareloParacatu; ${ }^{(10)}$ Latossolo Vermelho-Amarelo-Patrocínio; ${ }^{(11)}$ Ataque sulfúrico (Embrapa, 1997); e ${ }^{(12)}$ Composição mineralógica Método da alocação (Resende et al., 1987).

A CTSL é proposta como propriedade físico-química que define a resistência do solo à passagem de $\mathrm{S}$ da SRL para a SLL+SNL. O aumento da CTSL indica que o solo tem elevada reatividade por sulfato. De modo geral, verificaram-se aumentos consistentes da CTSL desde 30 d de incubação nos solos, exceto no RQ-CV, LV-TM e LVA-UB2, indicando dinâmica em favor da SLL+SNL (Quadro 4).No RQ-CV, desde $30 \mathrm{~d}$, a CTSL diminuiu de 0,152 para $0,098 \mathrm{mg} \mathrm{dm}^{-3} / \mathrm{mg} \mathrm{dm}^{-3}$, consequentemente maior resistência à saída de $S$ da SRL. No LV-TM, ao contrário do RQ-CV, ocorreu redução da CTSL de 0,179 para $0,087 \mathrm{mg} \mathrm{dm}^{-3} / \mathrm{mg} \mathrm{dm}^{-3}$ até $60 \mathrm{~d}$ de incubação, indicando resistência de passagem de $\mathrm{S}$ da SRL para a SLL+SNL. Aos $90 \mathrm{~d}$, observou-se aumento da CTSL, consequentemente houve redução da resistência da reserva SRL e gradiente a favor da SLL+SNL. No LVA-UB2, exceto aos 60 d, verificouse gradiente ao longo de todo período de incubação a favor da SLL+SNL, cuja CTSL aumentou de 0,148 para $0,425 \mathrm{mg} \mathrm{dm}^{-3} / \mathrm{mg} \mathrm{dm}^{-3}$. No LA-AR teve-se alternância de gradiente durante o período de incubação, sendo a resistência à saída de $\mathrm{S}$ da SRL preponderante.

A dinâmica entre as reservas dá ideia de um contínuo e fatores externos, como adubação e tempo de incubação (reatividade), podem deslocar o equilíbrio a favor de um ou outro reservatório, cuja magnitude depende das características intrínsecas dos solos. É provável que no início da incubação o aumento na concentração de $\mathrm{SO}_{4}{ }^{2-}$ na solução do solo promoveu dessorção de outros ânions retidos aos sítios de fixação, sendo essas cargas, então, ocupadas pelo $\mathrm{SO}_{4}{ }^{2-}$. A mudança na direção do gradiente entre os solos, ao longo do período de incubação, deve-se, provavelmente, à variação da reatividade do $\mathrm{SO}_{4}{ }^{2-}$ às cargas presentes na SLL+SNL, sendo esse deslocado por íon como o fosfato, que apresenta maior reatividade. Salim et al. (2004) defenderam a hipótese de continuidade entre os diferentes reservatórios, indicando que a relação entre o número de sítios de adsorção e a concentração dos íons em solução, a força de ligação entre óxidos e metais e o grau de saturação da solução são fatores que promovem a reversibilidade.

A elevada CTSL média observada nos solos LVAUB1, LVA-UB2, LV-SL, LVA-PR e LVA-PA é explicada pelos altos teores de óxidos de $\mathrm{Al}$ e Fe e consequentemente alta CMAS (Quadros 1 e 2), que favorecem a formação da reserva não lábil. Nesse caso, é possível que a reação do $\mathrm{S}$ ao metal forme um complexo monodentado de baixa reversibilidade (Evangelou, 1998). Particularmente no solo LVA-PA, de maior CTSL média, considera-se que a alta afinidade por fosfato, dada a sua capacidade máxima de adsorção de fósforo - CMAF, da ordem de 1.936 $\mathrm{mg} \mathrm{dm}^{-3}$ (Quadro 1), deve ter influenciado na dinâmica do $\mathrm{SO}_{4}{ }^{2-}$ nos $30 \mathrm{~d}$ iniciais da incubação, afetando seu gradiente a favor da reserva SLL+SNL. O aumento no tempo de incubação alterou essa dinâmica, favorecendo a fixação do $\mathrm{SO}_{4}{ }^{2-}$ em detrimento, provavelmente, do $\mathrm{PO}_{4}{ }^{2-}$. Observou-se que a reserva do SLL+SNL alcançou teor acima de $150 \mathrm{mg} \mathrm{dm}^{-3} \mathrm{de}$ S no final do período da incubação (Quadro 3, Figura 1). Pigna \& Violante (2003) e Mora et al. (2005) observaram que adsorção de $\mathrm{PO}_{4}$ foi influenciada pelo 


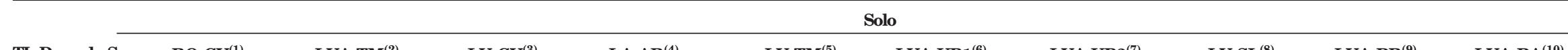

TI Dose des

SRL SLL+SNL SRL SLL+SNL SRL SLL+SNL SRL SLL+SNL SRL SLL+SNL SRL SLL+SNL SRL SLL+SNL SRL SLL+SNL SRL SLL+SNL SRL SLL+SNL

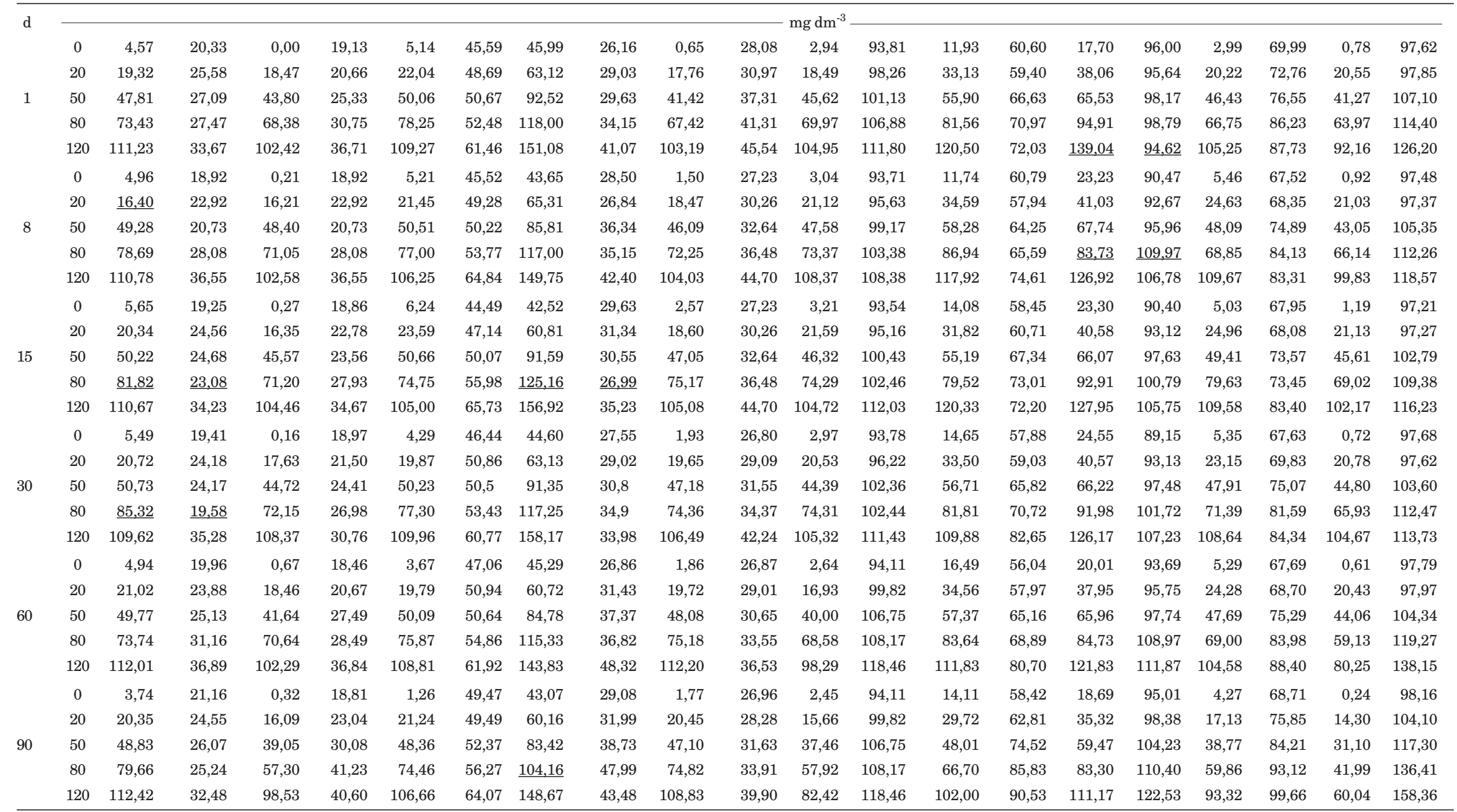

Os valores sublinhados não foram utilizados no ajuste das equações de SRL em função do SLL+SNL, por não representarem bem o fenômeno, uma vez que se trata de modelos preditivos e não explicativos. ${ }^{(1)}$ Neossolo Quartzarênico-Campina Verde; ${ }^{(2)}$ Latossolo Vermelho-Amarelo-Três Marias; ${ }^{(3)}$ Latossolo Vermelho-Campina Verde; ${ }^{(4)}$ Latossolo Amarelo-Aracruz; ${ }^{(5)}$ Latossolo Vermelho-Três Marias; ${ }^{(6)}$ Latossolo Vermelho-Amarelo-Uberaba (Amostra 1); ${ }^{(7)}$ Latossolo Vermelho-Amarelo-Uberaba (Amostra 2); ${ }^{(8)}$ Latossolo Vermelho-Sete Lagoas; ${ }^{(9)}$ Latossolo Vermelho-Amarelo-Paracatu; e ${ }^{(10)}$ Latossolo Vermelho-Amarelo-Patrocínio. 

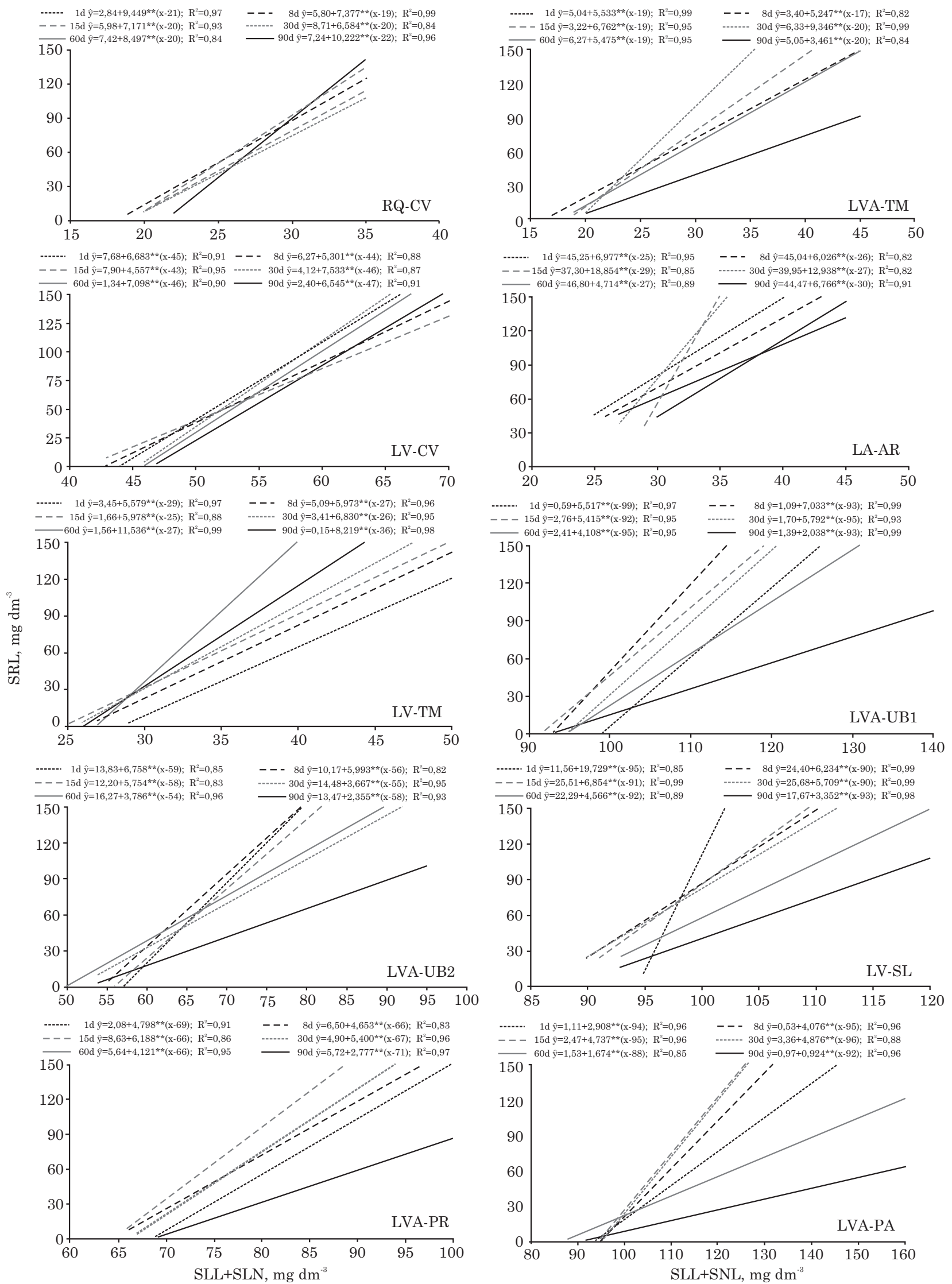

Figura 1. Relação entre os teores de SRL e os de SLL+SNL em diferentes tempos de incubação nos 10 solos estudados. ${ }^{* *}$ : significativo a $1 \%$. 
$\mathrm{SO}_{4}$. Metson \& Blackemore (1978) concluíram que a retenção de $\mathrm{SO}_{4}{ }^{2-}$ pelo solo, no que tange à competição com o fosfato, depende da quantidade relativa de $\mathrm{SO}_{4}{ }^{2-}$ na solução e da natureza do solo.

Ressalta-se o papel da matéria orgânica na formação do SRL e do SLL+SNL do LA-AR, LVA-UB1 e LVA-PA. A remoção da matéria orgânica aumentou a CMAS desses solos em $73 \%$ (64 para $111 \mathrm{mg} \mathrm{dm}^{-3}$ de S), $12 \%$ (188 para $211 \mathrm{mg} \mathrm{dm}^{-3}$ de S) e $25 \%$ (279 para $350 \mathrm{mg} \mathrm{dm}^{-3} \mathrm{de} \mathrm{S}$ ), respectivamente (Quadro 1), ao contrário do que ocorreu nos demais solos, cuja remoção da matéria orgânica diminuiu a CMAS. Esses resultados permitem postular que a matéria orgânica tem efeito destacado na microagregação e na estabilização dos microagregados dos Latossolos, reduzindo a sua superfície de contato; assim, sua remoção aumentou a superfície, proporcionado pela desestabilização dos microagregados, expondo sítios de carga mais ativos à adsorção de $\mathrm{SO}_{4}{ }^{2}$, especialmente da gibbsita, que tende a concentrar-se no núcleo dos microagregados (Schaefer, 2001). No LA-AR, pode-se supor que a remoção da matéria orgânica deve ter favorecido aumento de formas amorfas de caulinita ou ainda a exposição de núcleos de hematita, no interior de microagregados maiores, com elevada superfície específica e com alta CMAS. Dessa forma, a presença da matéria orgânica nesses solos diminuiu a CMAS e, consequentemente, o compartimento de SLL+SNL. Esse comportamento observado nesse estudo pode explicar os resultados contraditórios (Camps Arbestain et al., 2001) e inconclusivos (Kimsey Jr. et al., 2005) da matéria orgânica na retenção do $\mathrm{SO}_{4}{ }^{2-}$

No quadro 5, são apresentadas as classes de CTSL, os limites entre as classes e a classificação dos solos. A maioria dos solos evidenciou baixa CTSL, indicando que para cada $1 \mathrm{mg} \mathrm{dm}^{-3} \mathrm{de}$ SRL tem-se teor variando entre 0,119 e $0,2 \mathrm{mg} \mathrm{dm}^{-3} \mathrm{de}$ SLL+SNL.O solo LA-AR destacou-se por apresentar CTSL muito baixa (Quadro 5) e consequentemente maior reserva de SRL, superior a $130 \mathrm{mg} \mathrm{dm}^{-3}$, no maior tempo de incubação (Figura
1). No LA-AR, para cada $1 \mathrm{mg} \mathrm{dm}^{-3}$ de SRL tem-se $0,118 \mathrm{mg} \mathrm{dm}^{-3}$ de SLL+SNL, justificando sua classificação em muito baixa CTSL (Quadro 4). Esse comportamento pode ser por causa do domínio da caulinita na sua composição mineralógica (Quadro 2), cuja força de adsorção ao $\mathrm{SO}_{4}{ }^{2-}$ é relativamente baixa (Aylmore et al., 1967), tornando-o mais lábil.

As características químicas, físicas, físico-químicas e mineralógicas dos solos, dentro dos tempos de incubação, passaram a influenciar na CTSL, a partir dos $30 \mathrm{~d}$ de incubação (Quadro 6). Esse tempo foi necessário para o equilíbrio entre as reservas. Observou-se que tanto o $\mathrm{pH}$ quanto o teor de caulinita não apresentaram correlações significativas com a CTSL. A ausência de significância para o teor de caulinita nos solos, de modo geral, indicou a sua baixa eficiência na fixação de $\mathrm{SO}_{4}{ }^{2-}$, em relação aos óxidos de $\mathrm{Fe}$ e Al, descrita por Chao et al. (1964). Kimsey Jr. et al. (2005) também não observaram correlação entre pH e adsorção de sulfato em solos influenciados por cinzas vulcânicas. Nesse caso, é possível que a pouca variação em amplitude no $\mathrm{pH}$ dos solos justifique a ausência de correlação.

Já as correlações observadas entre a CTSL e os teores de C-orgânico, goethita e gibbsita dão indicações de que a formação da reserva SLL+SLN deve-se à fixação do $\mathrm{SO}_{4}{ }^{2-}$ às cargas geradas por essas frações (Quadro 6). A correlação significativa da CTSL com a CMAF indica que, possivelmente, as cargas que perfazem a CMAF não são específicas para o fosfato, podendo ser ocupadas pelo $\mathrm{SO}_{4}{ }^{2-}$. Embora seja presumido que o mecanismo eletrostático seja largamente responsável pela retenção de sulfato (Bohn et al., 2001), as evidencias indicam que houve dessorção de fosfato. O sulfato, assim como o fosfato, participa de reações de trocas de ligantes das superfícies de hidróxidos de $\mathrm{Fe}$ e $\mathrm{Al}$ (Rajan, 1979). Nessa condição, o sulfato adsorvido não é rapidamente dessorvido. Isso não significa que seja irreversível, indica que é necessário que outro fator desestabilize o sistema, como a adubação fosfatada.

Quadro 4. Capacidade-tampão do enxofre lábil (CTSL) determinada em seis tempos de incubação (TI) e CTSL média

\begin{tabular}{|c|c|c|c|c|c|c|c|c|c|c|}
\hline \multirow{2}{*}{ TI } & \multicolumn{10}{|c|}{ Solo } \\
\hline & RQ-CV & LVA-TM & LV-CV & LA-AR & LV-TM & LVA-UB1 & LVA-UB2 & LV-SL & LVA-PR & LVA-PA \\
\hline $\mathrm{d}$ & \multicolumn{10}{|c|}{$\mathrm{mg} \mathrm{dm} / 3 / \mathrm{mg} \mathrm{dm}^{-3}$} \\
\hline 1 & 0,118 & 0,181 & 0,150 & 0,143 & 0,179 & 0,175 & 0,148 & 0,051 & 0,208 & 0,344 \\
\hline 8 & 0,136 & 0,191 & 0,199 & 0,166 & 0,167 & 0,142 & 0,167 & 0,160 & 0,215 & 0,245 \\
\hline 15 & 0,139 & 0,148 & 0,219 & 0,053 & 0,167 & 0,185 & 0,174 & 0,147 & 0,162 & 0,211 \\
\hline 30 & 0,152 & 0,106 & 0,132 & 0,077 & 0,146 & 0,173 & 0,273 & 0,175 & 0,185 & 0,205 \\
\hline 60 & 0,118 & 0,183 & 0,141 & 0,121 & 0,087 & 0,244 & 0,264 & 0,219 & 0,243 & 0,597 \\
\hline 90 & 0,098 & 0,289 & 0,153 & 0,148 & 0,122 & 0,491 & 0,425 & 0,298 & 0,360 & 1,082 \\
\hline Média & 0,126 & 0,183 & 0,166 & 0,118 & 0,145 & 0,235 & 0,241 & 0,175 & 0,228 & 0,447 \\
\hline
\end{tabular}


Quadro 5. Classes de capacidade-tampão do enxofre lábil (CTSL) e classificação dos solos

\begin{tabular}{|c|c|c|c|c|}
\hline \multicolumn{5}{|c|}{ Classe de CTSL } \\
\hline Muito Baixa & Baixa & Média & Alta & Muito Alta \\
\hline$\leq 0,118$ & $0,119-0,200$ & $0,201-0,282$ & $0,283-0,364$ & $\geq 0,364$ \\
\hline LA-AR & RQ-CV; LVA-TM;LV-CV; LV-TM; LV-SL & LVA-UB1; LVA-UB2; LVA-PR; & - & LVA-PA \\
\hline
\end{tabular}

Quadro 6. Coeficientes de correlação linear simples entre a CTSL e algumas características químicas, físicas, físico-químicas e mineralógicas dos 10 solos, em diferentes tempos de incubação

\begin{tabular}{ccccccccccccc}
\hline $\mathbf{T I}^{(\mathbf{1})}$ & $\mathbf{p H}$ & $\mathbf{P - r e m}$ & $\mathbf{C O}^{(\mathbf{( 2 )}}$ & $\mathbf{C M A S}_{\mathbf{1}}^{(\mathbf{4})}$ & $\mathbf{C M A S}_{\mathbf{2}}{ }^{(\mathbf{5})}$ & $\mathbf{C M A F}^{(\mathbf{6})}$ & $\mathbf{D s}^{(\mathbf{7})}$ & $\mathbf{A}^{(\mathbf{8})}$ & $\mathbf{C t}^{(\mathbf{9 )}}$ & $\mathbf{G b}^{(\mathbf{1 0})}$ & $\mathbf{G t}^{(\mathbf{1 1})}$ & $\mathbf{K i}$ \\
\hline 1 & 0,05 & $-0,19$ & 0,05 & 0,39 & $0,67^{*}$ & 0,24 & $-0,11$ & $-0,06$ & 0,10 & $0,51^{\circ}$ & 0,36 & $-0,53^{\circ}$ \\
8 & $-0,22$ & $-0,35$ & 0,23 & $0,50^{\circ}$ & $0,57^{*}$ & 0,29 & $-0,21$ & 0,18 & 0,16 & 0,30 & $0,49^{\circ}$ & $-0,37$ \\
15 & 0,40 & $-0,39$ & 0,35 & 0,34 & 0,22 & 0,38 & $-0,32$ & 0,11 & $-0,17$ & 0,40 & $0,49^{\circ}$ & $-0,40$ \\
30 & 0,42 & $-0,70^{*}$ & $0,69^{*}$ & $0,65^{*}$ & $0,48^{\circ}$ & $0,73^{* *}$ & $-0,73^{* *}$ & $0,65^{*}$ & 0,09 & $0,62^{*}$ & $0,48^{\circ}$ & $-0,71^{* *}$ \\
60 & $-0,15$ & $-0,64^{*}$ & $0,58^{*}$ & $0,80^{* *}$ & $0,91^{* * *}$ & $0,66^{*}$ & $-0,55^{*}$ & $0,50^{\circ}$ & 0,11 & $0,66^{*}$ & $0,77^{* *}$ & $-0,57^{*}$ \\
90 & $-0,26$ & $-0,77^{* *}$ & $0,86^{* *}$ & $0,87^{* * *}$ & $0,77^{* *}$ & $0,80^{* *}$ & $-0,70^{*}$ & $0,66^{*}$ & 0,06 & $0,65^{*}$ & $0,93^{* * *}$ & $-0,55^{\circ}$ \\
\hline
\end{tabular}

(1) Tempo de incubação (d); ${ }^{(2)}$ P-rem: fósforo remanescente $\left(\mathrm{mg} \mathrm{L}^{-1}\right) ;{ }^{(3)} \mathrm{CO}$ : carbono orgânico (dag kg $\left.{ }^{-1}\right)$; ${ }^{(4)} \mathrm{CMAS}_{1}$ : capacidade máxima de adsorção de sulfato $\left(\mathrm{mg} \mathrm{dm}^{-3} \mathrm{de} \mathrm{S}\right) ;{ }^{(5)} \mathrm{CMAS}_{2}$ : capacidade máxima de adsorção de sulfato nos solos, após o ataque com $\mathrm{H}_{2} \mathrm{O}_{2} 30$ vol. (mg dm${ }^{-3}$ de S); ${ }^{(6)}$ CMAF: capacidade máxima de adsorção de fosfato $\left(\mathrm{mg} \mathrm{dm}^{-3}\right.$ de P); ${ }^{(7)}$ Ds: densidade do solo (kg dm $\left.{ }^{-3}\right)$;

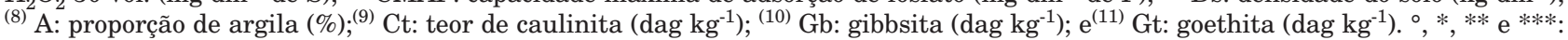
significativo a $10,5,1$ e $0,1 \%$, respectivamente.

\section{CONCLUSÕES}

1. O tempo de incubação e a adição de $\mathrm{S}$ aos solos, na forma de sulfato de cálcio, são fatores que determinam alteração na dinâmica do $\mathrm{S}$ entre as reservas de SRL e SLL+SNL.

2. O aumento do tempo de incubação (reatividade) favorece o movimento do $\mathrm{S}$ em direção a reserva do SLL+SNL, trazendo consequências diretas na disponibilidade desse nutriente às plantas.

3. A CTSL, variação da concentração de SLL+SNL em relação à variação da concentração de $\mathrm{SRL}(\mathrm{CTSL}=\Delta \mathrm{SLL}+\mathrm{SNL} / \Delta \mathrm{SRL})$, apresenta sensibilidade às mudanças de gradientes entre as reservas.

4. O aumento da CTSL dos solos relacionou-se, principalmente, com a capacidade máxima de adsorção de sulfato, teor de carbono orgânico e teores de goethita e gibbsita da composição mineralógica dos solos estudados.

\section{AGRADECIMENTOS}

À Coordenação de Aperfeiçoamento de Pessoal de Nível Superior (CAPES), ao CNPq pela concessão de bolsas de produtividade em pesquisa e a Pró-Reitoria de Pesquisa e Pós-Graduação da UFRR pelo suporte financeiro.

\section{LITERATURA CITADA}

ADAMS, F. \& RAWAJFIH, Z. Basaluminite and alunite: A possible cause of sulfate retention by acid soils. Soil Sci. Soc. Am. J., 41:686-692, 1977.

AJWA, H.A. \& TABATABAI, M.A. Metal-induced sulfate adsorption by soils: Effect of $\mathrm{pH}$ and ionic strength. Soil Sci., 159:32-42, 1995.

ALVAREZ V., V.H. Enxofre: Critérios de diagnose para o solo e planta, correção de deficiências e excessos. In: BORKET, C.M. \& LATMANN, A.F., eds. Enxofre e micronutrientes na agricultura brasileira. Londrina, Embrapa-CNPSo/ IAPAR/SBCS, 1988. p.31-59.

ALVAREZ V., V.H.; DIAS, L.E.; RIBEIRO Jr., E.S.; SOUZA, R.B. \& FONSECA, C.A. Métodos de análise de enxofre em solos e plantas. Viçosa, MG, Universidade Federal de Viçosa, 2001. 131p.

ALVAREZ V., V.H.; NOVAIS, R.F.; DIAS, L.E. \& OLIVEIRA, J.A. Determinação e uso do fósforo remanescente. B. Inf. SBCS, 25:27-32, 2000.

ALVES, M.E. \& LAVORENTI, A. Sulfate adsorption and its relationships with properties of representative soils of the Sao Paulo State, Brazil. Geoderma, 118:89-99, 2004.

AYLMORE, L.A.G.; KARIM, M. \& QUIRK, J.P. Adsorption and desorption of sulphate ions by soil constituents. Soil Sci., 103:10-15, 1967.

BARDSLEY, C.E. \& LANCASTER, J.D. Determinations of reserve sulfur and soluble sulfates in soils. Soil Sci. Soc. Am. Proc., 24:265-268, 1960. 
BARROW, N.J. Reactions with variable-charge soils. Dordrecht,Martinus Nijhoff Publ., 1987.

BARTON, D.; BILlETT, M.F.; CRESSER, M.S. \& HOPE, D. Sulphate adsorption capacity and $\mathrm{pH}$ of upland podzolic soils in Scotland: Effects of parent material, texture, and precipitation chemistry. Appl. Geochem., 9:127-139, 1994.

BARTON, D.; GAMMACK, S.M.; BILLETT, M.F. \& CRESSER, M.S. Sulphate adsorption and acidification of Calluna heathland and Scot pine forest Podzol soils in north-east Scotland. For. Ecol. Manage., 114:151$164,1999$.

BLAIR, G. Sulphur in tropics. Muscle Shoals, The Sulphur Institute, The International Fertilizer Development Center, 1979. 69p. (Technical Bulletin, T-12)

BOHN, H.L.; McNEAL, B.L. \& O’CONNOR, G.A. Soil chemistry. New York, John Wiley \& Sons, 2001.

CAMPS ABERSTAIN, M.; BARREAL, M.E. \& MACÍAS, F. Sulfate sorption in nonvolcanic Andisols and Andic soils from Galicia, NW Spain. Geoderma, 104:75-93, 2001.

CAMPS ABERSTAIN, M.; BARREAL, M.E. \& MACIAS, F. Phosphate and sulfate sorption in Spodosols with albic horizon from Northern Spain. Soil Sci. Soc. Am. J., 66:464$473,2002$.

CHAO, T.T.; HARWARD, M.E. \& FANG, S.C. Cationic effects on sulfate adsorption by soils. Soil Sci. Soc. Am. Proc., 28:632-635, 1964.

COUTO, W.; LATHWELL, D.J. \& BOULDIN, D.R. Sulfate sorption by Oxisols and an Alfisol of tropics. Soil Sci., 127:108-116, 1979.

DEFELIPO, B.V. \& RIBEIRO, A.C. Análise química do solo (metodologia). Viçosa, MG, Universidade Federal de Viçosa, 1981.17p. (Boletim Extensão, 29)

EMPRESA BRASILEIRA DE PESQUISA AGROPECUÁRIA EMBRAPA. Serviço Nacional de Levantamento e Conservação de Solos. Manual de métodos de análise de solo. 2.ed. Rio de Janeiro, 1997. 212p.

ENSMINGER, L.E. Some factors affecting the adsorption of sulfate by Alabama soils. Soil Sci. Soc. Am. Proc., 18:259$264,1954$.

EVANGELOU, V.P. Soil minerals and their surface properties.. In:EVANGELOU, V.P., ed.Environmental soil and water chemistry: Principles and applications.New York, John Wiley \& Sons, 1998. p.100-131.

FERNANDES, B. Retenção e movimento de água no solo. Viçosa, MG, Universidade Federal de Viçosa, 1967. 48p. (Dissertação de Mestrado)

HINGSTON, F.J.; POSNER, A.M. \& QUIRK, J.P. Anion adsorption by goethite and gibbsite. II. Desorption of anions from hydrous oxide surface. J. Soil. Sci., 25:16-26, 1974.
IONICS. Properties and characteristics of anion transfer membranes type 204-U386. Bulletin AR 204. 3-A. Ionics. Watertown, MA, 1987.

KIMSEYJr., M.; McDANIEL, P.; STRAWN, D. \& MOORE, J. Fate of applied sulfate in volcanic ash-influenced forest soils. Soil Sci. Soc. Am. J., 69:1507-1515, 2005.

METSON, A.J. \& BLACKEMORE, L.C. Sulphate retention by New Zealand soils in relation to the competitive effect of phosphate. New Zealand J. Agric. Res., 21:243-253, 1978.

MORA, M.D.L.; SHENE, C.; VIOLANTE, A.; DEMANET, R. \& BOLAN, N.S. The effects of organic matter and soil chemical properties on sulfate sorption in Chilean volcanic soils. In:Huang, P.M.; Violante, A.; Bollag, J.M. \& Vityakon, P., eds. Soil abiotic and biotic interactions and the impact on the ecosystem and human welfare. Enfield, Science Publication, 2005. p.223-244.

MOURA FILHO, W. Métodos de campo e laboratório. Viçosa, MG, Universidade Rural do Estado de Minas Gerais, 1964. 24 p.

NOVAIS, R.F \& SMYTH, T.J. Fósforo em solo e planta em condições tropicais. Viçosa, MG, Universidade Federal de Viçosa, 1999. 399p.

OLSEN, S.R. \& WATANABE, F.S. A method to determine a phosphorus adsorption maximun of soils as measured by the Langmuir Isotherm. Soil Sci. Soc. Am. Proc., 21:144-149, 1957.

PATIL, S.G.; SARMA, V.A.K. \& LOON, G.E. Acid rain, cation dissolution and sulphate retention in three tropical soils. J. Soil Sci., 40:85-93, 1989.

PIGNA, M. \& VIOLANTE, A. Adsorption of sulfate and phosphate on Andisols. Commun. Soil Sci. Plant Anal., 34:2099-2113, 2003.

RAJAN, S.S.S. Adsorption and desorption of sulfate and charge relationships in allophanic clays. Soil Sci. Soc. Am. J., 43:65-69, 1979.

RESENDE, M.; BAHIA FILHO, A.F.C. \& BRAGA, J.M. Mineralogia da argila de Latossolos estimada por alocação a partir do teor total de óxidos do ataque sulfúrico. R. Bras. Ci. Solo, 11:17-23, 1987.

SALIM, H.M.; GOBRAM, G.R.; GUAN, X. \& CLARKE, N. Mobility of sulfate in forest soils: Kinetic modeling. J. Environ. Qual., 33:488-495, 2004.

SCHAEFER, C.E.G.R.The B horizon microstructure of Brazilian Latosols as long-term biotic constructs. Aust. J. Soil Res., 39:909-926, 2001.

STRAHM, B.D. \& HARRISON, R.B.Mineral and organic matter controls on the sorption of macronutrient anions in variablecharge soils. Soil Sci. Soc. Am. J., 71:1926-1933, 2007.

UCHÔA, S.C.P.; ALVAREZ V., V.H.; FREIRE, F.M.; SCHAEFER, C.E.R. \& HARTMANN, L.K. Método de determinação de enxofre lábil com membrana de troca aniônica. R. Bras. Ci. Solo, 27:89-99, 2003. 